\title{
Copy number variant hotspots in Han Taiwanese population induced pluripotent stem cell lines - lessons from establishing the Taiwan human disease iPSC Consortium Bank
}

Ching-Ying Huang ${ }^{1}$, Ling-Hui Li', Wan-Tseng Hsu², Yu-Che Cheng ${ }^{1}$, Martin W. Nicholson', Chun-Lin Liu', Chien-Yu Ting ${ }^{1}$, Hui-Wen Ko ${ }^{3}$, Shih-Han Syu ${ }^{3}$, Cheng-Hao Wen ${ }^{3}$, Zhuge Yann ${ }^{4}$ Hsiang-Po Huang ${ }^{5}$, Hong-Lin Su', Po-Min Chiang ${ }^{7}$, Chia-Ning Shen ${ }^{8}$, Hsin-Fu Chen ${ }^{5}$, B. Lin Ju Yen ${ }^{9}$, Huai-En Lu', Shiaw-Min Hwang ${ }^{3}$, Shih-Hwa Chiou ${ }^{10}$, Hong-Nerng Ho ${ }^{11}$, Jer-Yuarn Wu ${ }^{1}$, Timothy J. Kamp ${ }^{12}$, Joseph C. Wu ${ }^{4}$ and Patrick C. H. Hsieh ${ }^{1 *}$ (D)

\begin{abstract}
Background: The Taiwan Human Disease iPSC Service Consortium was established to accelerate Taiwan's growing stem cell research initiatives and provide a platform for researchers interested in utilizing induced pluripotent stem cell (iPSC) technology. The consortium has generated and characterized 83 iPSC lines: 11 normal and 72 disease iPSC lines covering 21 different diseases, several of which are of high incidence in Taiwan. Whether there are any reprogramming-induced recurrent copy number variant (CNV) hotspots in iPSCs is still largely unknown.

Methods: We performed genome-wide copy number variant screening of 83 Han Taiwanese iPSC lines and compared them with 1093 control subjects using an Affymetrix genome-wide human SNP array.

Results: In the iPSCs, we identified ten specific CNV loci and seven "polymorphic" CNV regions that are associated with the reprogramming process. Additionally, we established several differentiation protocols for our iPSC lines. We demonstrated that our iPSC-derived cardiomyocytes respond to pharmacological agents and were successfully engrafted into the mouse myocardium demonstrating their potential application in cell therapy.

Conclusions: The CNV hotspots induced by cell reprogramming have successfully been identified in the current study. This finding may be used as a reference index for evaluating iPSC quality for future clinical applications. Our aim was to establish a national iPSC resource center generating iPSCs, made available to researchers, to benefit the stem cell community in Taiwan and throughout the world.
\end{abstract}

Keywords: Human induced pluripotent stem cell, Cell differentiation, Stem cell bank, Drug screening, Copy number variant, Hotspot

\footnotetext{
* Correspondence: phsieh@ibms.sinica.edu.tw

${ }^{1}$ Institute of Biomedical Sciences, Academia Sinica, Taipei 115, Taiwan

Full list of author information is available at the end of the article
}

\section{$\triangle B M C$}

(c) The Author(s). 2020 Open Access This article is licensed under a Creative Commons Attribution 4.0 International License, which permits use, sharing, adaptation, distribution and reproduction in any medium or format, as long as you give appropriate credit to the original author(s) and the source, provide a link to the Creative Commons licence, and indicate if changes were made. The images or other third party material in this article are included in the article's Creative Commons licence, unless indicated otherwise in a credit line to the material. If material is not included in the article's Creative Commons licence and your intended use is not permitted by statutory regulation or exceeds the permitted use, you will need to obtain permission directly from the copyright holder. To view a copy of this licence, visit http://creativecommons.org/licenses/by/4.0/ The Creative Commons Public Domain Dedication waiver (http://creativecommons.org/publicdomain/zero/1.0/) applies to the data made available in this article, unless otherwise stated in a credit line to the data. 


\section{Background}

In 2006, Yamanaka and his colleagues discovered that the combination of four specific transcription factors, Oct4, Sox-2, Klf4 and c-Myc, can reprogram mouse, and later human, fibroblasts into pluripotent stem cells capable of being reprogrammed into any cell type of the three germ layers. These newly derived cells were later termed induced pluripotent stem cells (iPSCs). With the possibility of differentiating iPSCs into different somatic cell types, the discovery holds great potential as a tool for studying human disease, drug development, and cell therapy $[1,2]$.

Over the past decade, there has been significant progress in realizing the potential of iPSC technology. Numerous iPSC-based studies have achieved significant breakthroughs in understanding human diseases and have begun translating research from the laboratory to the clinic, for example, in the treatment of age-related macular degeneration, spinal cord injury, and type 1 diabetes [3-5]. Despite iPSC translational studies in clinical trials, genome stability and the associated downstream problems, such as tumorigenesis, remain a key issue. Single nucleotide variations (SNV), structural variation such as copy number variations (CNV) or loss of heterozygosity (LOH), which may be inherited from donor cells, generated during the reprogramming process, or prolonged culture constrain the usage of iPSCs in basic research and clinical applications. Indeed, the world's first iPSC-based treatment for age-related macular degeneration was initially cancelled upon the discovery of SNVs and CNVs in the patient's iPSCs raising concerns about tumorigenicity $[5,6]$.

Further studies have shown that $\mathrm{CNV}$ amplification of the 20q11.21 region is the most recurrent hotspot in iPSCs and embryonic stem cells [7-10]. The genes encompassed by this region include anti-apoptotic genes, inhibitor of DNA binding 1 (ID1), BCL2-like1, and DNMT3B which is associated with pluripotency [11]. Moreover, by using high-resolution array comparative genomic hybridization to identify the unique CNV signatures for human iPSCs, Martins-Taylor et al. (2011) found that more than $25 \%$ of human iPSCs possessed recurrent CNVs at 1q31.3 and 17q21, and a human iPSCspecific CNV deletion at 8q24.3. Differences in the occurrence of genetic variation such as single nucleotide polymorphisms (SNPs) and CNV have been reported across ethnic groups, which may result in phenotypic variation and/or disease susceptibility [12]. However, whether there is a reprograming-induced genetic variation signature in iPSCs is still largely unknown.

"Han Chinese" is the largest ethnic group in the world; representing a unique population with different genetic backgrounds compared to others in the world, and most of Han Taiwanese are of Han Chinese descent. The Han
Taiwanese population is particularly unique due to the diversity of its gene pool which, in part, has arisen from colonizers such as the Dutch, Portuguese and Japanese. Over the past few years, several countries have established iPSC core facilities with the aim of improving the consistency and standardization of generating new iPSC lines [13-15]. In 2015, five institutes came together to meet an unmet need in Taiwan and formed the Taiwan Human Disease iPSC Service Consortium. This project sought to establish the first, validated, high-quality iPSC bank from healthy individuals and patients in Taiwan with the aim to develop therapies that effectively and exclusively target Chinese populations. Another objective was to employ our iPSC lines to address whether there is any genetic variation associated with reprogramming. At present, the project has generated 83 iPSC lines consisting of 11 normal iPSC lines from healthy donors and 72 iPSC lines covering 21 types of diseases. These lines span inherited diseases, chromosomal disorders, heart disease, neurodegenerative disease, neuropsychiatric disorders and rare diseases such as Fabry disease and facioscapulohumeral muscular dystrophy. We provide systematically derived and comprehensively characterized iPSC lines generated using our standardized operation protocols. To this end, each cell line was fully characterized for pluripotency and the presence of de novo CNVs. When comparing de novo CNVs in our iPSC lines with 1093 control subjects, we identified novel hotspots for recurrent $\mathrm{CNVs}$ during the reprogramming process. Additionally, we demonstrated the ability of our normal iPSC lines to differentiate into various somatic cell types, as well as a set of normal and disease iPSCs to specifically differentiate into cardiomyocytes (CM). Furthermore, using normal CMs, we demonstrate pharmacological responses to both isoproterenol and propranolol. These cells were also successfully engrafted in vivo.

To increase the utility of these valuable resources, both normal and disease iPSCs and the results of their characterization are available from the Bioresource Collection and Research Center (http://bcrc.firdi.org.tw/). We hope that sharing our experience in establishing this iPSC bank and generating quality-controlled iPSCs to benefit future efforts in basic and clinical research of the local and international stem cell research community.

\section{Methods}

\section{Reprogramming of donor cells}

Donor recruitment was approved by the Institutional Review Board of Biomedical Science Research at Academia Sinica (approval number AS-IRB02-106154 and ASIRB02-105099). All samples were collected from donors who agreed, by signed consent, to tissue donation and iPSC derivation. The reprogramming experiment was 
modified from a standard procedure of iPSC reprogramming [2] using CytoTune-iPS 2.0 Sendai Reprogramming Kit (Thermo Fisher Scientific).

\section{Detection of Sendai virus vectors and pluripotent gene expression}

To ensure the removal of Sendai virus and exogenous transgenes, and expression of endogenous pluripotent markers in iPSCs, total RNA was extracted from cells of greater than 10 passages using TRIzol reagent (Invitrogen). Then reversed transcription was performed by RevertAidTM H Minus First Strand cDNA Synthesis Kit (Fermentas). The primer sets are listed in Table S1.

\section{Immunofluorescence staining for pluripotency markers}

The PSC4-Marker Immunocytochemistry Kit (Life Technologies, Invitrogen) was used to analyze iPSC pluripotent marker expression. Following fixation and permeabilization, cells were stained with primary antibodies against OCT4, SOX2, SSEA-4 and TRA-1-60 and secondary antibodies with Alexa 594-and Alexa 488-conjugated for red and green fluorescence, respectively (Molecular Probes).

\section{Embryoid body formation assay}

Embryoid body formation was used to confirm differentiation potential into the three germ layers in vitro. $\mathrm{Hu}-$ man iPSCs were cultured in DMEM/F12 supplemented with $20 \% \mathrm{FBS}$ in ultra-low attachment 6-well plates (Corning) for 7 days after which embryoid bodies were re-plated onto $0.1 \%$ gelatin-coated plates. Fourteen days after re-plating, cells were then fixed with $4 \%$ formaldehyde and stained with antibodies against ectodermal marker $\beta$ III-Tubulin (TUJI), mesodermal marker $\alpha$ SMA, and endodermal marker, AFP (3-Germ Layer Immunocytochemistry Kit, Thermo Fisher Scientific).

\section{Teratoma formation assay}

Cells $\left(1 \times 10^{6}\right)$ were dissociated, re-suspended in 50\% Matrigel (Corning) and then transplanted into the testis of NOD/SCID mice. Mice were sacrificed on week 8 after transplantation. The teratomas were harvested, fixed and then embedded in paraffin for serial sectioning and histological analysis by haemotoxylin and eosin staining to confirm differentiation potential into different germ layers.

\section{Karyotyping}

Center for Medical Genetics of Changhua Christian Hospital in Taiwan performed karyotyping analysis. In order to induce cell cycle arrest and nuclear swelling, cells were treated with $10 \mu \mathrm{g} / \mathrm{mL}$ of Colcemid and 0.075 $\mathrm{M}$ hypotonic $\mathrm{KCl}$. The samples were then fixed with Carnoy's fixative. Metaphase chromosomes were harvested and subjected to Giemsa staining for cytogenic analysis of G-bands.

\section{In vitro cardiac toxicity assay}

Eighty thousand human iPSC derived CMs were treated with various dosages of doxorubicin, ranging from $1 \mu \mathrm{M}$ to $0.1 \mathrm{M}$ (Selleckchem) for $24 \mathrm{~h}$. Cell viability was assessed by Trypan Blue exclusion assay (Sigma) and cells were counted using TetraZ cell counting kits (BioLegend). The terminal deoxynucleotidyl transferase dUTP-mediated nick-end labeling (TUNEL) method was used to detect apoptotic cells after doxorubicin treatment. ApopTag Fluorescein in situ apoptosis detection kit was used as recommended by the manufacturer (Millipore).

\section{Cell engraftment assay}

Human iPSC derived CMs were used for cell engraftment assay after 20 days of CM differentiation. $1 \times 10^{6}$ CMs were suspended in fetal bovine serum (Gibco) supplemented with $100 \mathrm{ng} / \mathrm{mL}$ IGF1 (Peprotech) and 0.1\% of hyaluronic acid (Creative PEGWorks). Cells were injected into immunodeficient (SCID) mice; mice were sacrificed two weeks after transplantation. The graft was immunohistochemically confirmed with anti-human mitochondria antibody (Millipore, \#MAB1273) and antiTroponin I antibody (Genetex, GTX28289).

\section{Genomic DNA extraction}

Genomic DNA was prepared using the DNeasy Blood \& Tissue Kit (Qiagen) from each iPSC line and PBMCs as recommended by the manufacturer.

\section{Whole genome sequencing}

For Illumina library preparation, double-stranded DNA was quantified with a Qubit fluorescence assay (Life Technologies). The genomic DNA was sheared with a Covaris S2 instrument. Next Generation Sequencing library preparation was carried out using the TruSeq PCR free DNA HT kit (Illumina), essentially following manufacturer's manual. Individual DNA libraries size and concentration were measured using an Agilent 2100 bioanalyzer (Agilent), qPCR and Qubit (Life Technologies). Libraries were normalized to $4 \mathrm{nM}$ and stored at $20^{\circ} \mathrm{C}$ until use. For clustering and sequencing, normalized DNA libraries were combined into 5- or 6-sample pools per flowcell in all 8 lines and clustered on a cBot cluster instrument with paired-end cluster kit V4. All flowcells were sequenced on the Illumina HiSeq2500 sequencer using SBS kit V4 chemistry. In this study, we adopted the cloud-based DNA-seq analytics platform, SeqsLab (Atgenomix), to implement and accelerate the WGS best practice pipelines including data preprocessing, calling, annotation and interpretation of sequence variants [16]. Sequencing paired-end reads were 
mapped to the hg19 assembly of the human genome reference by using the BWA-MEM v.0.7.15 alignment algorithm [17]. Then we applied marking duplicates, pursuing local realignment, recalibrating base quality scores. The alignment files (BAM) generated from the data pre-processing were used with the following variant calling methods. SNVs and short indels were detected based on the GATK v.3.7 WGS Best Practices workflow by recalibrating base quality scores and performing indel realignment prior to SNV and indel calling, and recalibrating variant quality scores after variant calling. For structural variation discovery, we adopted DELLY2 v.0.7.6, which combines paired-end mapping information and split-read analysis for the discovery of balanced and unbalanced forms of structural variation, i.e., deletions, duplications, inversions, insertion and translocations, achieving high sensitivity and specificity throughout the genome. For somatic mutation discovery, we used GATK v.3.7 MuTect2, a somatic-specific genotyping tool with high sensitivity and specificity, for calling somatic SNVs and indels along with using COSMIC codingmutations database v.77 in conjunction with dbSNP b147 database to adjust the threshold for evidence of the variants in the parental cells. For variant annotation, many popular databases were curated in SeqsLab, the databases were categorized into several groups such as population, genomic context, clinical context, and functional context [16]. Common variants were defined as those with a frequency $>1 \%$ in the population database (e.g., The 1000 Genomes, ExAC, HapMap); rare variants were defined as those with frequency $<1 \%$. dbNSFP v.3.0 was used for annotation of functional perdition. Protein length change was defined as indels located in the exon.

\section{Healthy control subjects}

The 1093 control subjects were chosen from the Han Chinese Cell and Genome Bank (HCCGB) in Taiwan. All subjects received a physical check-up and questionnaire screening to exclude abnormal physical conditions and mental illness [18]. These individuals' CNV results served as controls in the CNV hotspot identification study.

\section{CNV hotspots and polymorphic CNV identification}

Affymetrix genome-wide human SNP array 6.0 (Affymetrix) was used for genome-wide CNV screening. The experiment was conducted according to the manufacturer's instruction by the National Center for Genome Medicine (Academia Sinica, Taiwan). All samples passed genotype quality control and genotype and copy number state of each probe was called using Affymetrix Genotyping Console software v.4.1. Sample identity was confirmed by matching the genotype data of the iPSC lines and their parental cell samples. Regions that contained at least ten consecutive probes with the same direction of copy number change were defined as having CNVs. We filtered out centromeric regions (hg19, UCSC), antibody variable regions, $\mathrm{T}$ cell receptor loci, pseudoautosomal regions and $\mathrm{X}$-transposed-region. In this study, only $\mathrm{CNV}$ regions larger than $100 \mathrm{~kb}$ were included for further analysis. Paired analysis mode in Genotyping Console software was applied to identify iPSC-specific CNVs. The output was manually curated. CNVs identified in iPSC and overlapped more than 50\% with any CNV region identified in their parental cells were excluded in order to eliminate inherited CNVs. To determine the frequency of iPSC-specific $\mathrm{CNV}$ regions identified in this study in the general population, iPSC-specific CNV loci were compared to control subjects. Loci that overlapped $<10 \%$ with CNVs in control subjects were included for analysis. iPSC-specific $\mathrm{CNV}$ hotspots were defined as the frequency of the CNVs in this study higher than $5 \%$ in the iPSC samples but not in the germline cells and rare in control subject group $(<0.2 \%)$. Likelihood Ratio Chi-square test was used to compare the difference in the CNV region between the iPSC sample and the control group, and a Bonferroni correction was used to adjust the $P$ value. $P \leq 0.00017$ is considered as significant.

To identify polymorphic CNV regions with frequent rearrangment, $\mathrm{CNV}$ regions were first identified in either parental cells or iPSC lines. Those regions with a frequency of occurrence $>5 \%$ in the total samples were selected. Using the Likelihood Ratio Chi-square test, the $\mathrm{CNV}$ regions present in parental cells and iPSCs were compared to 1093 control subjects. Subsequently, the $\mathrm{CNV}$ regions were manually examined to determine whether the region was only present in either the parental cells or paired iPSC line from all individuals. A CNV region was defined as a polymorphic $\mathrm{CNV}$ with frequent rearrangement if the mutually exclusive events were identified in more than 10 subjects.

For $\mathrm{CNV}$ burden assay, paired t-test was used to determine the association of total $\mathrm{CNV}$ count with reprogramming. Annotation of genes located in the CNV regions was according to UCSC genes (NCBI37/hg19).

\section{Cardiac differentiation and purification}

iPSCs were detached using Accutase (Innovative Cell Technologies) and approximately $1 \times 10^{5}$ cells were replated into Matrigel-coated 6 well plates. Once cells reached $\sim 80 \%$ confluency, cells were treated with $6 \mu \mathrm{M}$ CHIR99021 (Selleckchem) in RPMI/B27 insulin-free medium to induce mesoderm differentiation for $48 \mathrm{~h}$. Then, the culture medium was replaced with RPMI/B27 insulin-free medium for $24 \mathrm{~h}$. After that, cells were treated with $5 \mu \mathrm{M}$ IWR-1 (Sigma) for $48 \mathrm{~h}$. At day 5 , the medium was changed to remove IWR-1. Cells were then cultured in RPMI/B27 medium at day 7. Thereafter the medium was changed every other day until cells started 
to beat. Cardiac purification was performed through glucose starvation; cells were cultured in glucose-free RPMI/B27 for 4 days to increase the purity of cardiomyocytes.

\section{Endothelial cell differentiation}

To generate hiPSC-derived endothelial cells, we implemented a growth factor based protocol according to previous report [19].

\section{Neural induction and neuronal maturation}

When iPSCs reach $80 \%$ confluence, cells were treated with $1 \mathrm{mg} / \mathrm{ml}$ Dispase II (Roche) and harvested by scraping (Day 0). The detached cell clumps were dissociated into $200-300 \mu \mathrm{m}$ clusters and transferred to non-coated Petri dishes for $48 \mathrm{~h}$. The differentiation medium was DMEM/F12 (Invitrogen) supplied with 20\% knockout serum replacement (KSR, Invitrogen), $1 \mathrm{mM}$, nonessential amino acids (NEAA, Invitrogen), $2 \mathrm{mM}$ glutamate (Invitrogen) and $100 \mu \mathrm{M} 2$-mercaptoethanol (Invitrogen). On Day 2 of differentiation, the cell culture medium was replaced to DMEM/F12 supplied with $1 \%$ $\mathrm{N} 2$ supplement (Invitrogen), $1 \mathrm{mM}$ NEAAs and $2 \mathrm{mM}$ glutamate. The BiSF induction factors, including $10 \mathrm{ng} /$ $\mathrm{mL}$ recombinant human FGF-2 (rh-FGF2, R\&D Systems), $10 \mu \mathrm{M}$ SB431542 (Sigma-Aldrich) and $0.5 \mu \mathrm{M}$ BIO (Sigma-Aldrich), were added on Day 2 for $48 \mathrm{~h}$. From Day 0, $10 \mu \mathrm{M}$ Y27632 (Cayman Chemical) was provided until the end of BiSF treatment. On Day 4, the neural induction medium was removed, and the cells were cultured in neurobasal medium (Invitrogen) with $1 \% \mathrm{~N} 2$ supplement and $10 \mathrm{ng} / \mathrm{mL}$ rh-FGF2. After neural induction, the cells were dissociated into small clumps by Accutase and seeded on 1\% Geltrex (Invitrogen)coated cell culture dishes for neural maturation. Following differentiation, NPC purity was determined by immunocytochemistry staining with Nestin antibody (Biolegend, \#839801).

\section{Pancreatic differentiation}

Human iPSCs were grown on a vitronectin-coated plate, and maintained in a chemically defined medium (CDM) supplemented with Activin A $(10 \mathrm{ng} / \mathrm{mL})$. CDM medium contains $250 \mathrm{~mL}$ DMEM/F12 (GIBCO) and $250 \mathrm{~mL}$ IMDM (GIBCO) mixed 1:1, $5 \mathrm{~mL}$ concentrated lipids (GIBCO), $20 \mathrm{uL}$ Mercapto-thio-Glycerol (Sigma), $350 \mathrm{uL}$ Insulin (ROCHE), 250uL Transferrin (ROCHE), $5 \mathrm{~mL}$ Pen/Strep (GIBCO), and $0.5 \mathrm{~g}$ Polyvinylalcohol (SIGM A). Differentiation was carried out by induced growing iPSCs in CDM-PVA + Activin-A (100 ng/mL), BMP4 (10 $\mathrm{ng} / \mathrm{mL})$, bFGF $(20 \mathrm{ng} / \mathrm{mL})$ and LY $(10 \mathrm{mM})$ (AFBLy). The CDM-PVA AFBLy cocktail was changed daily until Day 3. Thereafter, the differentiation method was according to $[20,21]$. Immunocytochemistry was further performed on iPSC derived cells with pancreatic and duodenal homeobox 1 antibody to confirm differentiation efficiency.

\section{Hepatic differentiation}

The step-wise method to produce hepatocytes initiates from differentiating human iPSCs into definitive endoderm, which subsequently patterns into anterior endoderm and further developed into hepatic endoderm. The differentiation method was according to [21, 22]. Hepatic cells were further matured to produce cells expressing the hepatic specific protein albumin (ALB). Hepatic differentiation efficiency was examined by immunocytochemistry staining with albumin antibody (R\&D System, \#MAB1455).

\section{Granulosa cell differentiation}

For granulosa cell differentiation, human iPSCs were manually split and plated onto ultra-low attachment multiwell plates (Corning) to form embryonic bodies (EBs). Cells were cultured in maintenance medium $(80 \%$ DMEM/F12, 20\% knockout serum replacement, $4 \mathrm{ng} / \mathrm{mL}$ bFGF, $0.1 \mathrm{mM}$-mercaptoethanol, and 1\% NEAA (Gibco)) for $48 \mathrm{~h}$. Cells were then treated with $6 \mathrm{ng} / \mathrm{mL}$ BMP4 (R\&D systems) for $24 \mathrm{~h}$. On day 3, cells were then cultured in $10 \mathrm{ng} / \mathrm{mL}$ BMP4, $6 \mathrm{ng} / \mathrm{mL}$ WNT3A, $6 \mathrm{ng} / \mathrm{mL}$ activin-A, and $5 \mathrm{ng} / \mathrm{mL}$ bFGF (R\&D Systems) for $72 \mathrm{~h}$. Then EB-derived mesoderm progenitors were transferred to gelatin-coated plates and incubated in the maintenance medium supplemented with $10 \mathrm{ng} / \mathrm{mL}$ BMP4, $5 \mathrm{ng} / \mathrm{mL}$ bFGF, and $25 \mathrm{ng} / \mathrm{mL}$ follistatin (R\&D Systems) for 6 days.

\section{Results}

Establishment of the Taiwan human disease iPSC service consortium

The Taiwan Human Disease iPSC Service Consortium was established in 2015 consisting of 5 different institutes: the Institute of Biomedical Sciences, Academia Sinica (AS-IBMS), National Taiwan University Hospital (NTUH), Taipei Veterans General Hospital (TVGH), the Food Industry Research and Development Institute (FIRDI), and the National Health Research Institutes (NHRI) (Fig. 1a) and is funded by the Ministry of Science and Technology (MOST) of Taiwan. Our objective is to develop a resource for researchers both in Taiwan and abroad to provide validated and fully characterized iPSC lines which represent the Han Taiwanese population. Here, we aim to share our experience in establishing the Taiwan Human Disease iPSC Service Consortium to build the consortium facility as a resource center in Taiwan. The iPSC consortium enrolls regional hospitals across Taiwan to help identify and collect donor samples with represented diseases that fit our selection criteria to build 
A

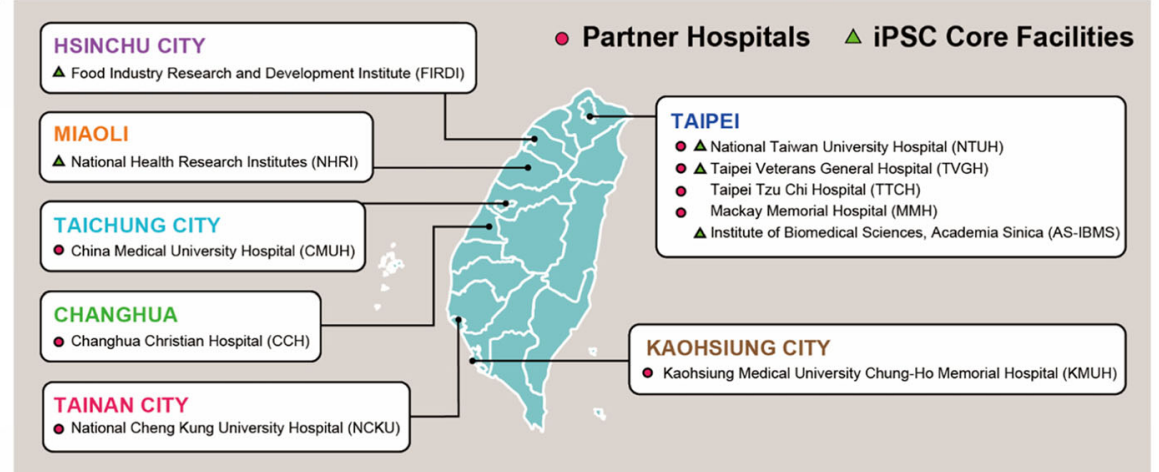

B

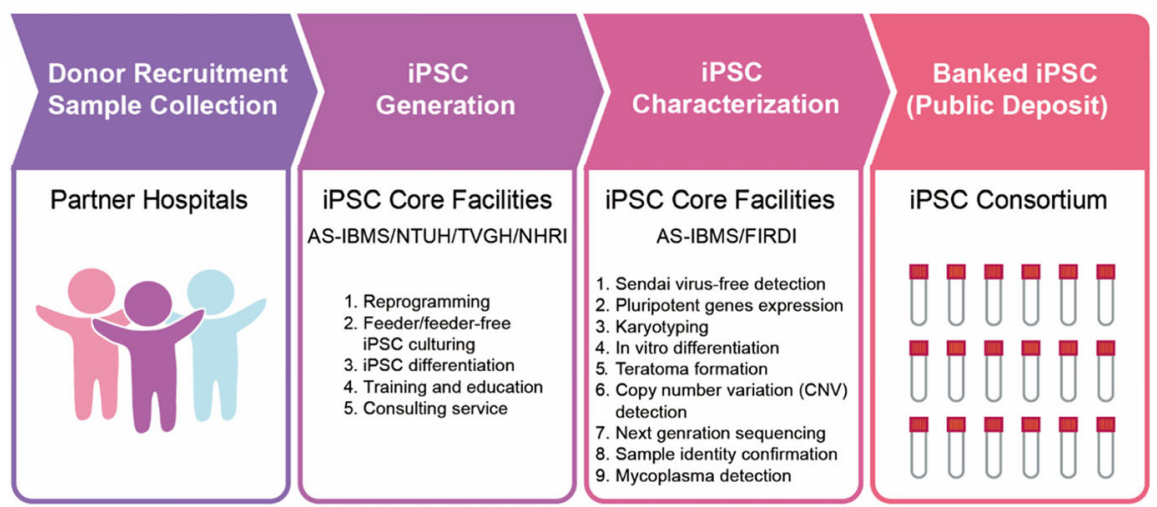

Fig. 1 Taiwan Human Disease iPSC Consortium Bank. a Map of Taiwan highlighting locations of iPSC core facilities and partner hospitals. b Workflow of the Taiwan Human Disease iPSC Service Consortium Bank

up the Taiwan iPSC bank. Our partner hospitals include eight different organizations located throughout Taiwan; National Taiwan University Hospital (NTUH), Taipei Veterans General Hospital (TVGH), Taipei Tzu Chi Hospital $(\mathrm{TTCH})$, Mackay Memorial Hospital (MMH), China Medical University Hospital (CMUH), Changhua Christian Hospital $(\mathrm{CCH})$, National Cheng Kung University Hospital (NCKUH), and Kaohsiung Medical University Chung-Ho Memorial Hospital (KMUH) (Fig. 1a).

\section{Generation, characterization and quality control testing of Han Taiwanese iPSC lines}

To ensure the consistency of the iPSC lines generated by the consortium, we established a standardized workflow across the network of sites within the consortium (Fig. 1b). All iPSC core facilities (IBMS, NTUH, TVGH and NHRI) follow a standardized protocol to handle patient samples and generate iPSCs. The Sendai virus reprogramming method has been reported as a time- and costeffective protocol for large-scale peripheral blood mononuclear cell (PBMC)- and fibroblasts -derived iPSC biobanking [23]. Thus, all iPSC lines are generated using Sendai virus and maintained in both feeder-dependent and independent culture systems. These are subsequently sent to a centralized facility, the Bioresource Collection and Research Center (BCRC) in the FIRDI, for comprehensive characterization including, sendai virus-free detection, pluripotent gene expression, karyotyping, in vitro differentiation, and in vivo teratoma formation. Clones that successfully passed the characterization criteria were then bio-banked (Fig. 1b; Fig. S1). The only exceptions are cell lines for Turner syndrome (NTUH-iPSC-004/45,X/46, $\mathrm{X}, \operatorname{idic}(\mathrm{X})(\mathrm{q} 22)$ ), which is characterized by an abnormality in the $\mathrm{X}$ chromosome, and a monogenic diabetes iPSC line (TVGH-iPSC-016; 46,XY,-16,+mar [20]) who's karyotypic abnormalities were inherited from the original donor cells (Fig. S2). Donors for the disease iPSC lines were selected based on the following criteria: patients with monogenic, polygenic, or chromosomally-inherited diseases or variants of unknown significance. In total, 76 Han Taiwanese donors were recruited, generating 83 lines. There were 11 normal lines and 72 disease lines, covering 21 diseases. Several of the disease lines carry mutations that are of high incidence in Taiwan. There were one to three patients for each disease type, with at least three clones generated for each patient. Donor gender, age, ethnicity, and known variants were also recorded. There were 43 females and 40 males in our iPSC bank (Table S2). These iPSC 
lines are available as a tool for research and development in Taiwan and abroad.

\section{Identification of genomic variation in normal Han Taiwanese iPSC lines}

We assessed the DNA integrity of our iPSCs by monitoring genomic variation events resulting from the reprogramming process. To this end, we conducted whole genome sequencing on ten pairs of our normal iPSC lines along with their parental cells. SeqsLab bioinformatics software (Atgenomix Inc., https://www.biorxiv. org/content/early/2017/12/27/239962) was used to streamline the genome sequencing secondary and ternary data analyses, adapted from GATK Best Practices and based on ACMG Standards and Guidelines for the interpretation of genomic variants. The data preprocessing, variant calling, variant annotation, and quality control workflows adapted are depicted in Fig. 2a. GATK3 HaplotypeCaller, DELLY2 and GATK3 MuTect2 were utilized to call different types of variants within these lines and their parental cells, such as SNVs, short insertions and deletions (indels), and structural variations. Somatic mutations were also evaluated by a Tumor-Normal variant calling method, where these iPSC lines were compared with their corresponding parental cells (PBMCs or fibroblasts) in an analogous manner to which tumor cells are compared to normal tissue to identify variants in tumor cells [24]. Variants with coverage of less than $25 \mathrm{X}$ and inherited variants were excluded from downstream analysis. GATK3 HaplotypeCaller was used to call SNV, short indels, and multinucleotide variations in ten pairs of normal iPSCparental cells. The number of iPSC-specific variants identified across all iPSC lines ranged from 3590 to 8127 with an average of 5497. Overall, the distribution of different types of variants called by GATK3 HaplotypeCaller among all samples were similar (Fig. 2b, and Table S3). Further, classification of these variants based on population allele frequency, common variants were defined as those with a frequency $\geq 1 \%$ in the population database (The 1000 Genomes, ExAC, and HapMap); rare variants were defined as those with frequency $<1 \%$ in the population database. dbNSFP v.3.0 was used for annotation of functional perdition. Protein length change was defined as indels located in the exon. Classification of these variants based on population allele frequency, the locus in chromosomal regions, functional prediction, and sequence effect indicated no functional differences among samples (Fig. 2e). To investigate iPSC-specific pathogenic variants, GATK3 MuTect2 was utilized in accordance with the 2015 ACMG guidelines (Standards and Guidelines for the Interpretation of Sequence Variants). The number of variants identified using GATK3 MuTect2 Tumor-Normal pipeline across all iPSC lines ranged from 6523 to 7646 . Based on the same classification criteria above, a similar trend in variant distribution was found in that there was no functional difference among samples (Fig. 2d). Variants affecting coding DNA sequence and the encoded protein (indels in exonic regions) were found in a small number ranging from 3 to 9 in each iPSC line (Fig. 2g). Characterization and annotation of these indicated that none were tumorigenicrelated. DELLY2, a structural variant calling tool, was used to identify duplication, insertions, and deletions greater than $300 \mathrm{bp}$. The average number of iPSCspecific structural variants was 420 and was similar across all iPSC lines except for two iPSC lines which contained more structural variants (FIRDI-iPSC-002 and NHRI-iPSC-001). In general, the number of variants ranged from 241 to 1061 (Fig. 2c) and showed a uniform pattern in type and location (Fig. 2c and f). We also investigated whether there was any common genomic variation among these iPSC lines; we did not find any significant variation that was shared among the $10 \mathrm{iPSC}$ lines using the three different calling methods.

\section{Identification of CNV hotspots in Han Taiwanese iPSC lines using Affymetrix genome-wide human SNP Array}

To examine whether the reprogramming process could induce de novo $\mathrm{CNV}$ generation and to identify whether there are any recurrent $\mathrm{CNV}$ hotspots associated with the reprogramming process, we analyzed 83 iPSC lines and their parental cells using the Affymetrix GenomeWide Human SNP Array 6.0 which contains more than 906,600 probes for SNPs, and 945,826 probes for CNVs. All samples passed the genotyping quality control test (average sample call rate $=99.24 \%$ ). The pipeline for $\mathrm{CNV}$ filtering and annotation is summarized in Fig. 3a. $\mathrm{CNVs}$ that were either generated during or after the reprogramming process at autosomes and sex chromosomes were analyzed by comparing CNVs identified in iPSC lines and respective parental cells. We excluded all inherited CNVs and filtered out centromeric regions, antibody variable regions, T-cell receptor loci, pseudoautosomal regions and $\mathrm{X}$-transposed-region and Turner Syndrome samples. CNVs with length larger than $100 \mathrm{~kb}$ were included for further analysis. CNVs located in the same loci among samples were identified using $10 \%$ reciprocal overlap cutoff. In total, we identified 168 iPSCspecific CNV loci (Table S4) from 82 iPSC samples. The details of iPSC-specific CNV regions among various iPSC lines are listed in Table S5. Notably, the length of the majority (80.1\%) of CNVs was less than $500 \mathrm{~Kb}$, $15.3 \%$ of CNVs were $500 \mathrm{~Kb}-1 \mathrm{Mb}$ and $4.2 \%$ of CNVs were $1 \mathrm{Mb}-5 \mathrm{Mb}$. Only $2 \mathrm{CNVs}$ were larger than $5 \mathrm{Mb}$ (contained in NTUH-iPSC-010 and TVGH-iPSC-024 iPSC lines; Fig. 3b). All lines were assessed for CNV burden before and after reprogramming. There was no 


\section{A}

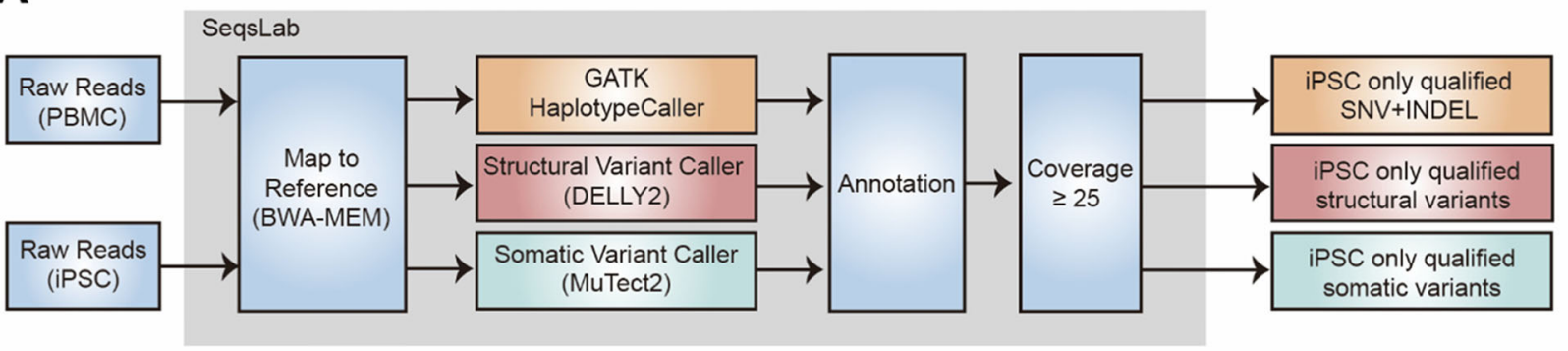

B

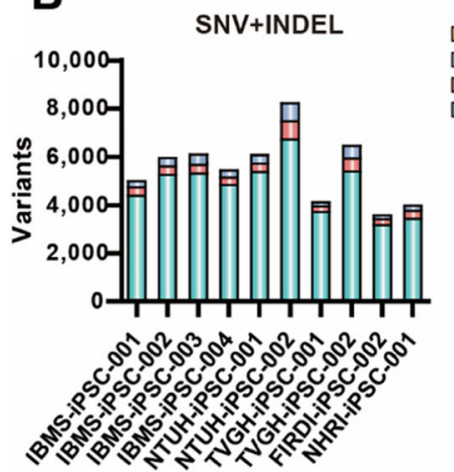

E

SNV+INDEL

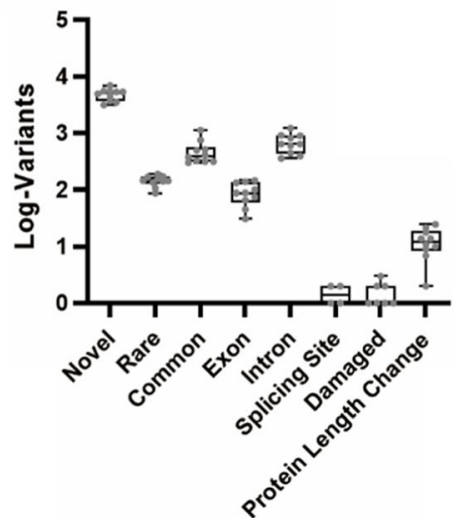

C

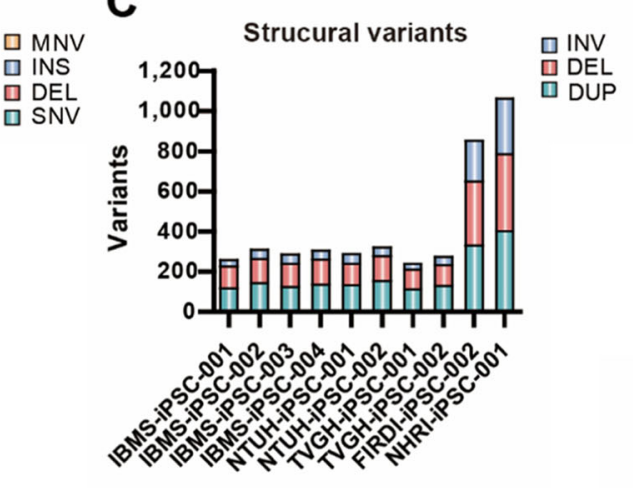

F

Structural variants

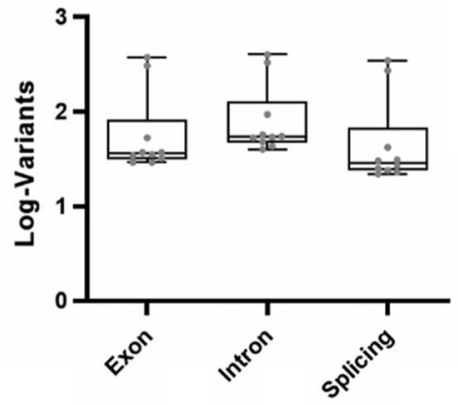

D

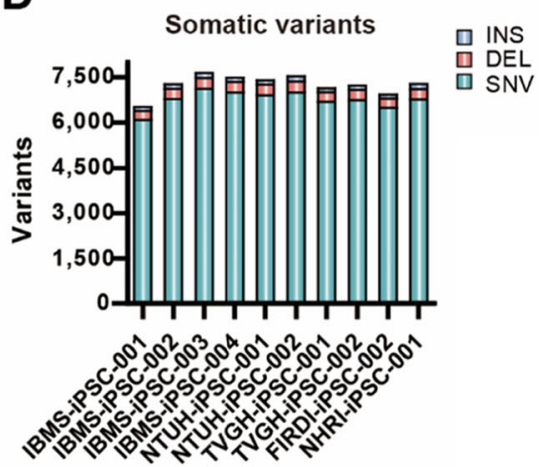

G

Somatic variants

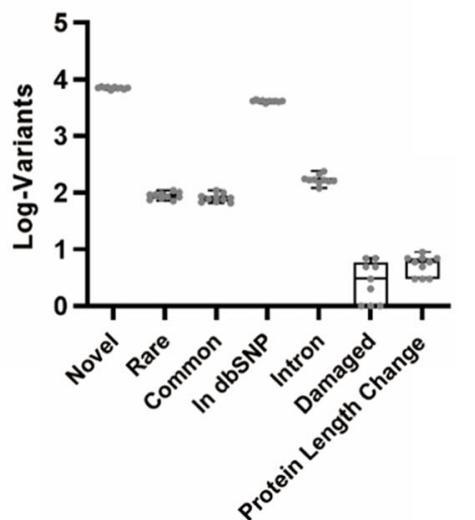

Fig. 2 Characterization of variants generated during reprogramming in 10 normal iPSC lines. a A flowchart describing the filtering strategy used to obtain SNVs, structural variants and somatic variants specific to reprogrammed cell line using GATK HaplotypeCaller, DELLY2 and MuTect2. b-d The number of single nucleotide variants (SNV), deletions (DEL), insertion (INS) and multi-nucleotide variations (MNV) of iPSC-specific qualified variants among iPSCs identified by $\mathbf{b}$ GATK HaplotypeCaller, $\mathbf{c}$ DELLY2, and $\mathbf{d}$ MuTect2. e-g Distribution of variant categories of iPSC-specific qualified variants among iPSCs identified by e GATK HaplotypeCaller, $\mathbf{f}$ DELLY2, and $\mathbf{g}$ MuTect2. Data in e, $\mathbf{f}$, and $\mathbf{g}$ are represented as mean \pm SEM

increased burden in the iPSC lines compared to their respective parental cells $(P=0.477$; Fig. 3c). To identify any iPSC-specific CNV hotspot regions associated with reprogramming, the rates of $\mathrm{CNV}$ at autosomes and sex chromosomes were compared between all iPSC lines and 1093 control subjects ( 525 males, 568 females) from Taiwan. CNV regions on autosomes were analyzed in all samples whereas $\mathrm{CNV}$ regions on sex chromosomes were analyzed by gender. iPSC-specific hotspots were defined as CNV regions with a frequency of occurrence $>5 \%$ in iPSC lines but not found in their paired parental cells and the rates of such $\mathrm{CNV}$ in control subjects were less than $0.2 \%$. These hotspots were significantly associated with reprogramming. We successfully identified 10 iPSC-specific CNV hotspots located at chromosomes 4, $5,6,7,10,13,20$ and X, with $91 \%$ of the CNV hotspots 
A
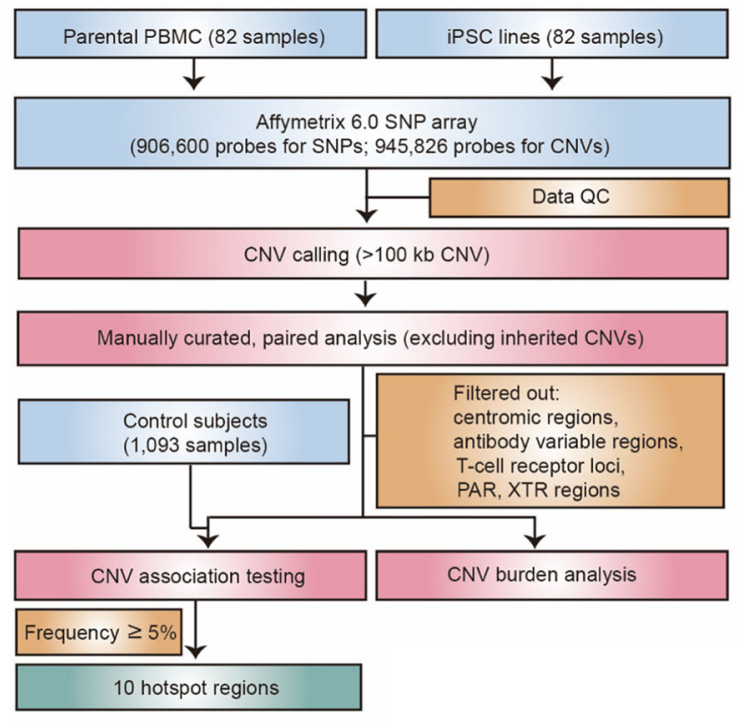

D

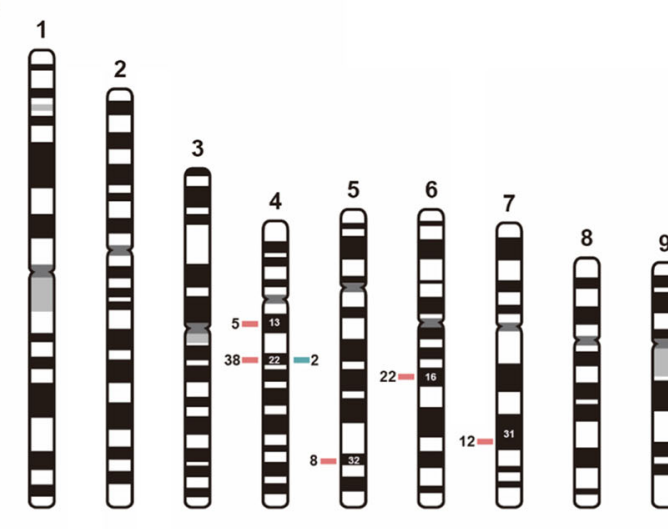

B

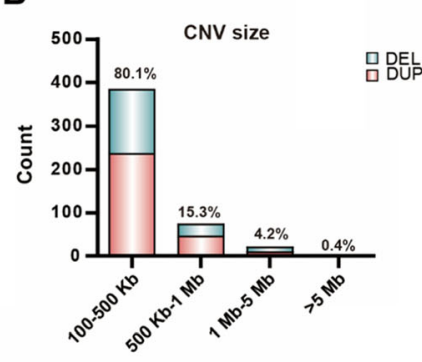

C

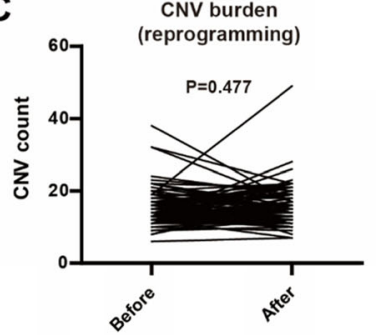

Fig. 3 Characteristics of CNVs induced by reprogramming. a Workflow of CNV identification. b Distribution of the size of CNVs among iPSCS induced by reprogramming. $\mathbf{c}$ CNV burden analysis of cells before and after iPSC reprogramming $\mathbf{d}$ Chromosomal distribution of rearrangement of hotspot regions of iPSC-specific CNVs from 82 iPSC lines

being duplications (Fig. 3d and Table 1). Most of the cell sources for reprogramming in our bank are PBMCs, as PBMCs are a heterogeneous mixture of cell types. To confirm whether these hotspots can be found in iPSC lines derived from fibroblasts, we analyzed all subclones of our fibroblast-derived iPSC lines (3 iPSC lines), and found that some fibroblast-derived subclones contain the same $\mathrm{CNV}$ hotspot regions. For example, duplication of Chr4q22.1-q22-2 was found in all 4 subclones of
IBMS-iPSC-031 and 3 subclones of TVGH-iPSC-025. Moreover, copy number gain within $\mathrm{ChrXq} 23$ was identified among all 4 IBMS-iPSC-031 subclones (Table S5). To determine if there are polymorphic CNV regions with frequent rearrangement during reprogramming, $\mathrm{CNV}$ regions with a frequency of occurrence $>5 \%$ in iPSCs and parental cells but not always existing in paired samples were selected. Using the Likelihood Ratio Chisquare test, the $\mathrm{CNV}$ regions present in parental cells 
Table 1 List of genes at the hotspot regions of iPSC-specific CNVs

\begin{tabular}{|c|c|c|c|c|c|c|c|c|c|}
\hline \multirow[b]{2}{*}{ Chr } & \multirow[b]{2}{*}{ Start } & \multirow[b]{2}{*}{ End } & \multirow[b]{2}{*}{ Locus } & \multicolumn{2}{|c|}{ iPSCs $(\boldsymbol{n}=82)$} & \multicolumn{2}{|c|}{ Control $(\boldsymbol{n}=1093)$} & \multirow[b]{2}{*}{$\boldsymbol{P}$ value } & \multirow[b]{2}{*}{ Genes Contained } \\
\hline & & & & Duplication & Deletion & Duplication & Deletion & & \\
\hline 4 & 62690392 & 62945462 & q13.1 & 5 & 0 & 1 & 0 & $<0.0001$ & ADGRL3, ADGRL3-AS1 \\
\hline 4 & 92930866 & 94220988 & $\begin{array}{l}\mathrm{q} 22.1- \\
\mathrm{q} 22-2\end{array}$ & 38 & 2 & 0 & 1 & $<0.0001$ & GRID2, LNCPRESS2 \\
\hline 5 & 147202104 & 147449067 & q32 & 8 & 0 & 0 & 0 & $<0.0001$ & SPINK5, SPINK1, SCGB3A2, C5orf46 \\
\hline 6 & 94291842 & 94849832 & q16.1 & 22 & 0 & 0 & 0 & $<0.0001$ & TSG1 \\
\hline 7 & 121549706 & 122245008 & q31.32 & 12 & 0 & 1 & 0 & $<0.0001$ & PTPRZ1, CADPS2, AASS, FEZF1-AS1, FEZF1 \\
\hline 10 & 54932474 & 55398702 & q21.1 & 6 & 0 & 0 & 0 & $<0.0001$ & NA \\
\hline 13 & 55695571 & 56471149 & q21.1 & 15 & 0 & 0 & 2 & $<0.0001$ & MIR5007 \\
\hline 20 & 29620219 & 31558271 & $q 11.21$ & 5 & 0 & 0 & 0 & $<0.0001$ & $\begin{array}{l}\text { MYLK2, EFCAB8, BCL2L1, HM13, FRG1BP, FRG1DP, } \\
\text { DEFB122, COMMD7, POFUT1, NOL4L, HCK, PDRG1, } \\
\text { NOL4L-DT, MLLT10P1, DEFB115, DEFB116, DEFB119, } \\
\text { DEFB118, DEFB121, DEFB124, DEFB123, LINC00028, } \\
\text { REM1, ID1, HM13-AS1, MIR3193, COX4I2, TPX2, ABA- } \\
\text { LON, DUSP15, FOXS1, TTLL9, MIR7641-2, CCM2L, } \\
\text { XKR7, PLAGL2, TM9SF4, TSPY26P, KIF3B, MIR1825, } \\
\text { ASXL1, LOC101929698, C20orf203, DNMT3B, MAPRE1 }\end{array}$ \\
\hline$x$ & 112880475 & 113795367 & q23 & 27 & 0 & 0 & 0 & $<0.0001$ & XACT \\
\hline$x$ & 114766295 & 114897324 & q23 & 8 & 0 & 0 & 0 & $<0.0001$ & PLS3,PLS3-AS1 \\
\hline
\end{tabular}

* For CNVs at X-chromosome, we only compare female iPSC $(n=42)$ and female controls $(n=568)$

and iPSCs were compared to the 1093 control subjects. Seven CNV regions (chromosome 1, 4, 7, 8, 14, 15 and 16) were identified as common within the iPSCs or parental cells, and control group $(\mathrm{P}>0.05)$. The data showed that these $7 \mathrm{CNV}$ regions are "polymorphic" $\mathrm{CNV}$ regions in this population, and suggested that rearrangement of these polymorphic CNV regions occurred frequently during the reprogramming process. Detailed information about these polymorphic CNV regions is listed in Table S6. These results indicated that the reprogramming process induced rearrangement of $10 \mathrm{CNV}$ hotspot regions and 7 polymorphic CNV regions in the general population of Taiwan. When using the functional annotation tool DAVID for clustering of genes located within CNV hotspots and polymorphic CNVs, the result revealed that most genes were clustered in signaling peptide, secreted, and extracellular regions. We did not identify any genes within these regions which have been associated with tumorigenesis.

\section{Derivation of iPSCs into different somatic lineages}

To assess the utility of our iPSC lines, we differentiated at least two of our normal iPSC lines into various somatic cell types, such as retinal pigment epithelium, neural progenitor cells, cardiomyocytes (CM), hepatocytes, pancreatic cells, endothelial cells and granulosa cells. Immunofluorescence was employed to verify the expression of specific markers such as the retinal pigment epithelium marker RPE65, neural progenitor cell specific marker nestin, cardiac specific marker $\alpha$-actinin, hepatic cell specific marker albumin, $\beta$-cell precursor marker pancreatic and duodenal homeobox 1, and endothelial cell marker PECAM1, in their respective differentiated cell type (Fig. 4a). Quantification of cells expressing each marker is shown in Fig. 4b-g. iPSCs were also differentiated into germ-like cells, which showed mRNA expression of follicle-stimulating hormone receptor in the granulosa cell stage at day 12 and 14 after differentiation (Fig. 4h). Together, these data show that our cell lines have the potential to differentiate into multiple cell types.

\section{iPSC-derived cardiomyocytes respond to doxorubicin and} engraft into mouse myocardium

We next sought to determine whether our normal and disease iPSC lines could be used for disease modeling, drug screening and clinical use. To this end, we differentiated 11 normal and 7 disease iPSC lines into CMs. The percentage of troponin-I positive cells was confirmed by flow cytometry on day 20 after cardiac differentiation (Fig. 4i). Quantification of troponin-I positive cells across each iPSC line is shown in Fig. 4j. To verify appropriate drug responses, we first assessed the effects of either the $\beta$-adrenergic agonist isoproterenol or the beta-blocker propranolol. The iPSC-derived CMs showed a dose-dependent response to both drugs, namely, increased beating frequency upon treatment with isoproterenol and decreased beating frequency with propranolol (Fig. S3A). The $\mathrm{EC}_{50}$ for isoproterenol and propranolol were $0.37 \mu \mathrm{M}$ and $3.6 \mu \mathrm{M}$. We then tested the effect of $24 \mathrm{~h}$ exposure of increasing doses of doxorubicin, known to have cardiotoxic effects on CMs 


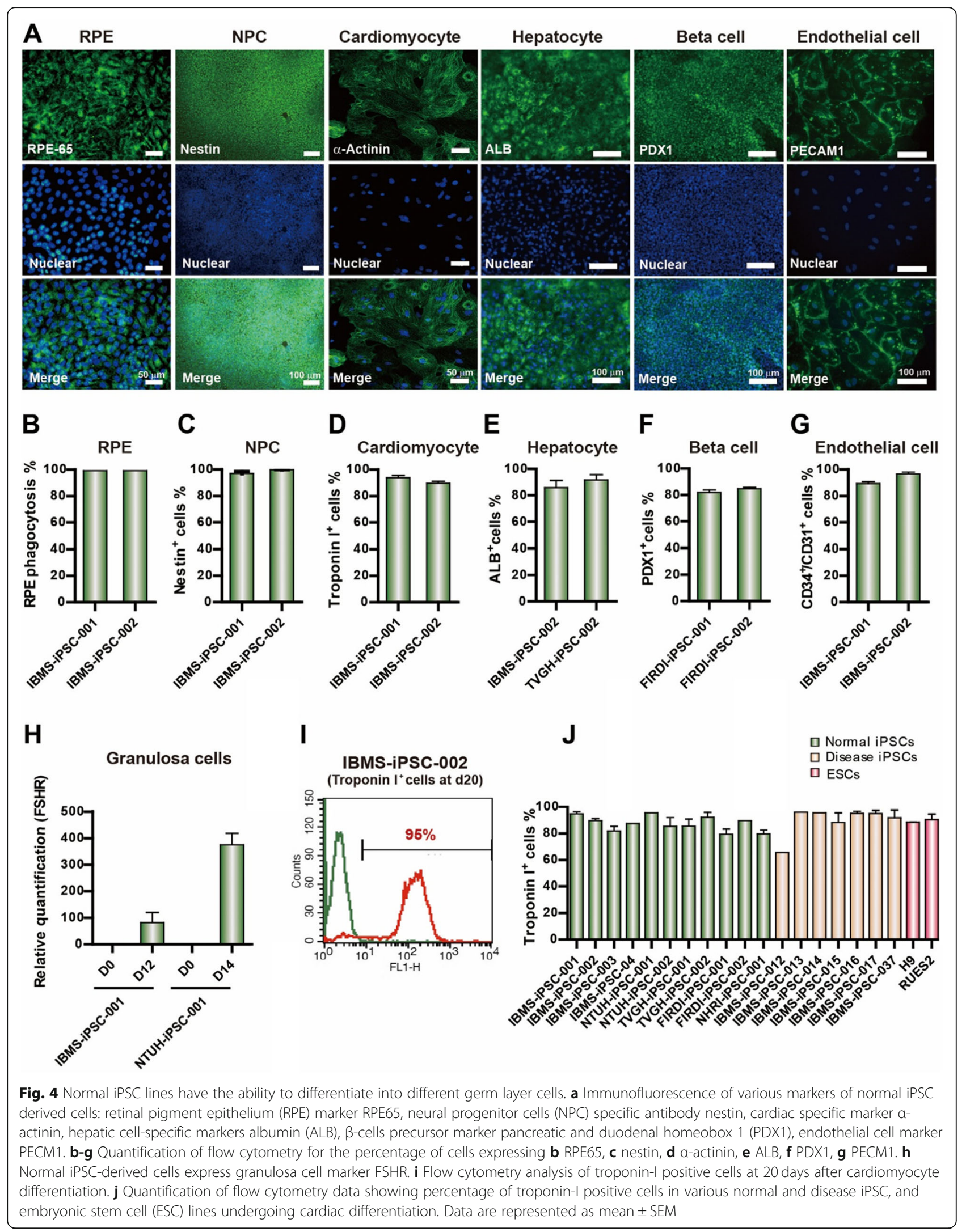


derived from two of our normal iPSC lines (IBMS-iPSC002 and FIRDI-iPSC-002). All CMs showed a dosedependent cardiotoxicity to doxorubicin with an IC50 of $1.9 \mu \mathrm{M}$ and $13.6 \mu \mathrm{M}$ (Fig. S3B). Furthermore, doxorubicin-induced cardiotoxicity was confirmed by TdT-mediated dUTP nick end-labeling (TUNEL) assay. TUNEL-positive cells were observed at $24 \mathrm{~h}$ after iPSCderived CMs were treated with $1 \mu \mathrm{M}$ doxorubicin (Fig. S3C). Finally, we also examined the ability of our iPSCderived $\mathrm{CMs}$ to engraft into the mouse myocardium through direct injection of $1 \times 10^{6}$ IBMS-iPSC-001-derived $\mathrm{CMs}$ into the myocardium of nude mice. A schematic diagram of the cell engraftment assay is shown in Fig. S3D. Cells that successfully engrafted were identified at 2 weeks post-injection using a human-specific mitochondrial antibody (Fig. S3E) whereas the surrounding myocardial area was identified through either $\alpha$-myosin heavy chain (MHC) or cardiac Troponin I antibodies. The results (Fig. S3E) showed clear engraftment of iPSC-derived CMs into the mouse myocardium which indicates the clinical potential of these cells.

\section{Discussion}

The current report details the experience of establishing a large-cohort iPSC bank representing the genetic background of the general population in Taiwan and the identification of CNV hotspots during the reprogramming process. Since its discovery, iPSC technology has become a powerful tool for understanding the correlation between patient genotype and phenotype and recently has shown promising clinical applications [25]. In order to provide high-quality fully characterized iPSCs, the Taiwan Human Disease iPSC Consortium Bank has developed strict standard operating protocols to generate and culture each iPSC line to ensure consistency across our four iPSC core facilities. All iPSC lines were generated using Sendai virus non-integrating method. iPSCs with ES-like morphology were selected and tested for existence of the Sendai virus. Subsequent to that, standard characterization of each line included examination of cell morphology, pluripotency assessment, karyotyping, and in vitro and in vivo differentiation. We also incorporated microbiology contamination testing, genome-wide SNP and CNV detection, and cell identity confirmation (STR-PCR and SNP genotyping) in our characterization protocol. Currently, the Taiwan iPSC bank has collected and generated more than 83 fully characterized normal and disease iPSC lines spanning 21 diseases. Our iPSC bank recruited patients carrying monogenic and polygenic disorders; variants of unknown significance, chromosomally-inherited diseases; and family-specific variation with several carrying mutations that are of high incidence in Taiwan. For example, the Taiwan Human Disease iPSC Consortium Bank holds iPSC lines which carry the $\alpha$-galactosidase mutation c.936 $+919 \mathrm{G}>\mathrm{A}$ in Fabry disease patients. Additionally, G2385R polymorphism of the LRRK2 gene which is a known risk factor variant for Parkinson's Disease (Cai et al., 2013) in the Han Chinese population, as well as the aldehyde dehydrogenases-2 SNP rs671, which occurs in approximately $45 \%$ of the population in Taiwan [22, 26-28]. Together, this bank holds high quality, fully characterized iPSC lines which represent the local Han Taiwanese population in Taiwan.

Previous reports have found that most CNVs are generated during early passage after cell reprogramming [15]. Due to budget scale, we used the highly costeffective Affymetrix Genome-Wide Human SNP Array 6.0 to investigate genomic integrity. In agreement with previous studies, the overwhelming majority (97.9\%) of the CNVs detected in our iPSC lines (passage 12-20) were less than $2 \mathrm{Mb}$ [15]. Notably, in 8 iPSC lines, with an otherwise normal karyotype, our array data identified $10 \mathrm{CNVs}$ larger than $2 \mathrm{Mb}$ (2.1\%), with two larger than $5 \mathrm{Mb}$. These chromosomal abnormalities were excluded when performing G-banded karyotyping as G-banded karyotyping has a limited resolution to $5 \mathrm{Mb}-10 \mathrm{Mb}$ and the risk of overlooking chromosomal abnormalities is increased when karyotyping results give poor G-banding pattern (Schrock et al., 1996). Trisomies 8 and 12 are a common karyotypic abnormality found in iPSCs and embryonic stem cells as shown in a previous study [29]. In contrast, all of our iPSC lines displayed normal karyotype except for Turner syndrome iPSC lines and a monogenic diabetes iPSC line which was also found in the parental cells and thus not due to iPSC reprogramming.

Variations in iPSCs are predominantly inherited from their parental cells $[30,31]$. To highlight iPSC-specific $\mathrm{CNV}$ hotspots, we examined CNVs with frequency higher than 5\% and only found in the iPSCs but not in the paired parental cells. Ten of these iPSC-unique CNV regions were found, and furthermore, all of them were either rare or not found in the 1093 Taiwan general population control subjects (frequency $<0.2 \%$ ) (Table 1 ), suggesting that the reprogramming process induced these recurrent CNVs. Notably, most of the CNV hotspots we identified are copy number gain, which is unsurprising as most copy number deletions result in cell death. Intriguingly, we also identified polymorphic CNVs with frequent rearrangement during reprogramming, evident by the occurrence or disappearance of these CNVs between paired iPSC and parental cells. These results not only suggest that these CNVs might be associated with recombination hotspots during cell proliferation, but also these CNVs might be unstable from generation to generation in the population due to the frequent rearrangement nature. 
Consistent with data from [32], we found that the distribution of reprogramming-induced CNVs is nonrandom as nearly $48.8 \%$ of our iPSC lines have copy number gains and losses within the Chr4q22.1-q22-2 region. This de novo CNV was also detected and verified using quantitative PCR analysis in retroviralreprogrammed iPSCs and Sendai virus-reprogrammed iPSCs in a previous study [33]. CNV hotspot regions on chromosome 5 (Chr5q32) and 7 (Chr7q31.32) have also been reported to be generated during reprogramming [34]. In 2018, Popp et al. found that a mosaic gain in Chr20q11.21 represented a possible hotspot CNV region in two of their iPSC lines [11]. This result is consistent with our finding on duplication within Chr20q11.21 region. Genes located in the hotspot region Chr20q11.21 (ID1, BCL2L1 and HM13) were found to have minimal amplicon in ESC lines in a previous study, and BCL2L1 has been shown as a candidate for lead adaptive benefits for ESC culture [35]. Furthermore, a long noncoding RNA, XACT located in ChrXq23 duplication hotspots, has been re-expressed in human iPSCs to control human $\mathrm{X}$-chromosome inactivation initiation, whereas, XACT was silenced in iPSC-derived mesenchymal stem cells $[33,36]$. This result suggests that CNV hotspots may have functional significance in iPSCs. When using DAVI $\mathrm{D}$ for functional annotation clustering of genes located within CNV hotspots, we did not find genes within these regions associated with tumorigenesis. Although we successfully identified ten CNV hotspots strongly associated with reprogramming using genome-wide SNP array, we note that there are still some technical limitations. Hybridization intensity-based $\mathrm{CNV}$ inference is unable to detect balanced translocations and inversions. The sensitivity of SNP array does not support the detection of low-level chromosomal mosaicism. Finally, uneven distribution of SNP probes affects the CNV evaluation. Hence, for complete characterization when surveying genomic integrity of iPSCs, both genome-wide analysis and G-banded karyotyping should be included.

\section{Conclusions}

In summary, iPSC generation and characterization are time-consuming, expensive, and labor-intensive process. We hereby present the establishment of the first iPSC core in Taiwan, representing genetically, the general Han Taiwanese population. The implementation of standardized protocols for iPSC generation and characterization enabled consistent production of high-quality iPSCs. Several iPSC lines were obtained from normal, healthy subjects, as well as numerous iPSC lines from patients with a spectrum of representative diseases. Furthermore, the CNV hotspots induced by cell reprogramming have successfully been identified in the current study. Whether evaluation of genetic variation should be included in iPSC characterization remains unclear, however, this finding may be used as a reference index for evaluating iPSC quality for future clinical applications. We also expect that the CNV hotspots identified in this study can help to establish characterization standards for iPSC genetic integrity. Overall, these items mirror issues discussed in previous international stem cell banking initiatives [37]. With the establishment of an easily-accessible service, systematic procedures, and well-organized repository, we envisage that the core facility and iPSC bank will become an invaluable resource for both public and private research.

\section{Supplementary information}

Supplementary information accompanies this paper at https://doi.org/10. 1186/s12929-020-00682-7.

Additional file 1. Figure S1. Characterization of iPSC cell line IBMSiPSC-002-07. (A) Reverse transcription-polymerase chain reaction (RT-PCR) analyses of Sendai-virus (SEV) and human KLF4, KLF4- OCT3/4-SOX2

(KOS), C-MYC, and GAPDH . (B) RT-PCR analyses of ESC-marker expression in the iPSC line. (C) Immunofluorescence analyses of stemness marker expression. (D) In vitro differentiation of iPSCs into the three different germlayers by embryonic formation assay. (E) Histological staining of teratoma derived from a normal iPSC line; N: neuronal structure, G: glandular structure; C: cartilage-like structure. (F) Representative G-banded chromosomes. Karyotypes of normal iPSCs. Figure S2 Karyotype analysis of the iPSC lines. (A) Karyotype of premature ovarian failure iPSC, NTUH-iPSC004-06 (Turner disease). (B) Karyotype of parental cells of TVGH-iPSC-016, arrow indicates rearranged chromosome 16. (C) Karyotype of TVGH-iPSC016 (monogenic diabetes iPSC), arrow indicates missing chromosome 16. Figure S3 In vitro and in vivo functional evaluation of iPSC derived cardiomyocytes. (A) Beating frequency (beats/min) of iPSC-derived cardiomyocytes under isoproterenol or propranolol treatment. (B) The doseresponse relationship for doxorubicin (DOX) treatment of iPSC derived cardiomyocytes, as evaluated by TetraZ cell counting assay. (C) TUNEL assay showing cell death $24 \mathrm{~h}$ after doxorubicin treatment. (D) Workflow of iPSC-CM engraftment in the mouse heart. (E) Immunostaining of the mouse myocardium engrafted with derived from human iPSC, showing engrafted of human iPSC-CMs. h-Mito: anti-human mitochondria antibody. Data are represented as mean \pm SEM.

Additional file 2. Table S1. Primer list for RT-PCR. Table S2. A List of Available Normal and Disease iPSCs in the Taiwan Disease iPSC Service Consortium Cell Bank. Table S3. The number of SNV, DEL, INS and MNV of iPSC-specific qualified variants among iPSCs identified by GATK HaplotypeCaller. Table S4. List of iPSC-specific CNV loci. Table S5. Summary of iPSC-specific CNV loci among various iPSC lines. Table S6. List of genes at the "polymorphic" CNV regions strongly associated with the reprogramming process

\section{Abbreviations}

AS-IBMS: Institute of Biomedical Sciences, Academia Sinica; BCRC: Bioresource Collection and Research Center; $\mathrm{CCH}$ : Changhua Christian Hospital;

CM: Cardiomyocyte; CMUH: China Medical University Hospital; CNV: Copy number variation; FIRDI: Food Industry Research and Development Institute; HCCGB: Han Chinese Cell and Genome Bank; iPSC: Induced pluripotent stem cell; KMUH: Kaohsiung Medical University Chung-Ho Memorial Hospital; LOH: Loss of heterozygosity; Indels: Short insertions and deletions; MHC: Myosin heavy chain; MMH: Mackay Memorial Hospital; NCKUH: National Cheng Kung University Hospital; NHRI: National Health Research Institutes; NTUH: National Taiwan University Hospital; PBMC: Peripheral blood mononuclear cell; RPE: Retinal pigment epithelial cell; SNP: Single nucleotide polymorphism; SNV: Single nucleotide variations; TTCH: Taipei Tzu Chi Hospital; TUNEL: TdT-mediated dUTP nick end-labeling assay; TVGH: Taipei Veterans General Hospital 


\section{Acknowledgements}

We also thank the Taiwan Mouse Clinic and the National Center for Genome Medicine for the technical support. We thank Dr. Chung-Tsai Su and Dr. Ming-Tai Chang for proposing the flow for data analysis, using SegsLab to process all of NGS analysis pipeline and consolidate the experimental results for discussion. We also thank Dr. Yuan Tsong Chen for providing healthy controls' SNP genotyping result and Chen-Zen Lo for providing the technical support on CNV analysis.

\section{Authors' contributions}

P.C.H. conceived the project and designed the study; Y.C.C., C.L.L., C.Y.T., H.P.H., and L.Y. generated the iPSCS. H.E.L, H.W.K, S.H.S, C.H.W. and S.M.H. characterized and banked the IPSC, L.H.L. and C.H.Y performed CNV data analyzing and interpretation. W.T.H. performed cardiac toxicity experiments and analyzed data. M.W.N., H.N.L, and H.L.S generated iPSC-derived neural progenitor cells. P.M.C. generated iPSC-derived endothelial cells. C.N.S. generated iPSC-derived hepatocytes and pancreatic cells. H.F.C and H.N.H. generated iPSC-derived granulosa cells. S.H.C. generated iPSC-derived retinal pigment epithelium. C.Y.H. and Z.Y. generated IPSC-derived cardiomyocytes. M.W.N. helped for English editing of the paper. C.Y.H. managed the study and wrote the paper in consultation with T.J.K. and J.W. All authors discussed the results and commented on the manuscript. The author(s) read and approved the final manuscript.

\section{Funding}

The Consortium and this study are funded by the Ministry of Science and Technology (MOST) (MOST 107-2319-B-001-003, MOST 108-2319-B-001-004, MOST 107-2321-B-001-029, and MOST 108-2321-B-001-017, MOST 109-2740B-001-002).

\section{Availability of data and materials}

The information for normal/disease iPSC lines are available in Taiwan iPSC Consortium Cell Bank (http://ipsc.ibms.sinica.edu.tw/index.html; https:// catalog.bcrc.firdi.org.tw/Welcome). All iPSC lines in this study are readily available from the BCRC cell bank (https://catalog.bcrc.firdi.org.tw/Welcome). The datasets generated during and/or analysed during the current study are available from the corresponding author on reasonable request.

\section{Ethics approval and consent to participate}

Donor recruitment was approved by the Institutional Review Board of Biomedical Science Research at Academia Sinica (approval number ASIRB02-106154 and AS-IRB02-105099).

\section{Consent for publication}

Not applicable.

\section{Competing interests}

The authors declare that they have no competing interests.

\section{Author details}

'Institute of Biomedical Sciences, Academia Sinica, Taipei 115, Taiwan. ${ }^{2}$ School of Pharmacy, College of Medicine, National Taiwan University, Taipei 100, Taiwan. ${ }^{3}$ Bioresource Collection and Research Center, Food Industry Research and Development Institute, Hsinchu 300, Taiwan. ${ }^{4}$ Stanford Cardiovascular Institute, Stanford University School of Medicine, Stanford, CA 94305, USA. ${ }^{5}$ Graduate Institute of Medical Genomics and Proteomics, College of Medicine, National Taiwan University, Taipei 100, Taiwan. ${ }^{6}$ Department of Life Sciences, National Chung-Hsing University, Taichung 402, Taiwan. ${ }^{7}$ Institute of Clinical Medicine, National Cheng Kung University, Tainan 701, Taiwan. ${ }^{8}$ Genomics Research Center, Academia Sinica, Taipei 115 , Taiwan. ${ }^{9}$ Institute of Cellular and System Medicine, National Health Research Institutes, Zhunan 350, Taiwan. ${ }^{10}$ Institute of Pharmacology, School of Medicine, National Yang-Ming University, Taipei 112, Taiwan. ${ }^{11}$ Department of Obstetrics and Gynecology, College of Medicine and the Hospital, National Taiwan University, Taipei, Taiwan. ${ }^{12}$ Stem Cell and Regenerative Medicine Center, University of Wisconsin-Madison, Madison, WI 53705, USA.
Received: 2 April 2020 Accepted: 24 August 2020

Published online: 04 September 2020

\section{References}

1. Takahashi K, Yamanaka S. Induction of pluripotent stem cells from mouse embryonic and adult fibroblast cultures by defined factors. Cell. 2006;126(4): 663-76.

2. Takahashi K, Tanabe K, Ohnuki M, Narita M, Ichisaka T, Tomoda K, Yamanaka S. Induction of pluripotent stem cells from adult human fibroblasts by defined factors. Cell. 2007;131(5):861-72.

3. Trounson A, McDonald C. Stem cell therapies in clinical trials: Progress and challenges. Cell Stem Cell. 2015;17(1):11-22.

4. Trounson A, DeWitt ND. Pluripotent stem cells progressing to the clinic. Nat Rev Mol Cell Biol. 2016;17(3):194-200.

5. Mandai M, Kurimoto $Y$, Takahashi M. Autologous induced stem-cell-derived retinal cells for macular degeneration. N Engl J Med. 2017;377(8):792-3.

6. Garber K. RIKEN suspends first clinical trial involving induced pluripotent stem cells. Nat Biotechnol. 2015;33(9):890-1.

7. Elliott AM, Elliott KA, Kammesheidt A. High resolution array-CGH characterization of human stem cells using a stem cell focused microarray. Mol Biotechnol. 2010;46(3):234-42.

8. Martins-Taylor K, Nisler BS, Taapken SM, Compton T, Crandall L, Montgomery KD, Lalande $\mathrm{M}, \mathrm{Xu} \mathrm{RH}$. Recurrent copy number variations in human induced pluripotent stem cells. Nat Biotechnol. 2011;29(6):488-91.

9. Laurent LC, Ulitsky I, Slavin I, Tran H, Schork A, Morey R, Lynch C, Harness JV, Lee S, Barrero MJ, et al. Dynamic changes in the copy number of pluripotency and cell proliferation genes in human ESCs and iPSCs during reprogramming and time in culture. Cell Stem Cell. 2011:8(1):106-18.

10. Salomonis N, Dexheimer PJ, Omberg L, Schroll R, Bush S, Huo J, Schriml L, Ho Sui S, Keddache M, Mayhew C, et al. Integrated genomic analysis of diverse induced pluripotent stem cells from the progenitor cell biology consortium. Stem cell reports. 2016;7(1):110-25.

11. Popp B, Krumbiegel M, Grosch J, Sommer A, Uebe S, Kohl Z, Plotz S, Farrell M, Trautmann U, Kraus C, et al. Need for high-resolution genetic analysis in iPSC: results and lessons from the ForlPS consortium. Sci Rep. 2018;8.

12. White SJ, Vissers LE, Geurts van Kessel A, de Menezes RX, Kalay E, Lehesjoki $A E$, Giordano $\mathrm{PC}$, van de Vosse $\mathrm{E}$, Breuning $\mathrm{MH}$, Brunner $\mathrm{HG}$, et al. Variation of CNV distribution in five different ethnic populations. Cytogenetic Genome Res. 2007;118(1):19-30.

13. McKernan R, Watt FM. What is the point of large-scale collections of human induced pluripotent stem cells? Nat Biotechnol. 2013;31(10):875-7.

14. De Sousa PA, Steeg R, Wachter E, Bruce K, King J, Hoeve M, Khadun S, McConnachie G, Holder J, Kurtz A, et al. Rapid establishment of the European Bank for induced pluripotent stem cells (EBiSC) - the hot start experience. Stem Cell Res. 2017;20:105-14.

15. Panopoulos AD, D'Antonio M, Benaglio P, Williams R, Hashem SI, Schuldt BM, DeBoever C, Arias AD, Garcia M, Nelson BC, et al. iPSCORE: a resource of 222 iPSC lines enabling functional characterization of genetic variation across a variety of cell types. Stem Cell Rep. 2017;8(4):1086-100.

16. Chang M-T, Tung Y-A, Chung J-M, Yao H-F, Li Y-L, Lin Y-H, Wang Y-T, Chen C-Y, Su C-T. SeqsLab: an integrated platform for cohort-based annotation and interpretation of genetic variants on spark; 2017.

17. Li H, Handsaker B, Wysoker A, Fennell T, Ruan J, Homer N, Marth G, Abecasis G, Durbin R. The Sequence Alignment/Map format and SAMtools. Bioinformatics (Oxford, England). 2009;25(16):2078-9.

18. Pan WH, Fann CS, Wu JY, Hung YT, Ho MS, Tai TH, Chen YJ, Liao CJ, Yang ML, Cheng AT, et al. Han Chinese cell and genome bank in Taiwan: purpose, design and ethical considerations. Hum Hered. 2006;61(1):27-30.

19. Wu YT, Yu IS, Tsai KJ, Shih CY, Hwang SM, Su IJ, Chiang PM. Defining minimum essential factors to derive highly pure human endothelial cells from iPS/ES cells in an animal substance-free system. Sci Rep. 2015;5:9718.

20. Bertero A, Pawlowski M, Ortmann D, Snijders K, Yiangou L, Cardoso de Brito M, Brown S, Bernard WG, Cooper JD, Giacomelli E, et al. Optimized inducible shRNA and CRISPR/Cas9 platforms for in vitro studies of human development using hPSCs. Development (Cambridge, England). 2016; 143(23):4405-18.

21. Cho CH, Hannan NR, Docherty FM, Docherty HM, Joao Lima M, Trotter MW, Docherty K, Vallier L. Inhibition of activin/nodal signalling is necessary for pancreatic differentiation of human pluripotent stem cells. Diabetologia. 2012:55(12):3284-95 
22. Cai J, Lin Y, Chen W, Lin Q, Cai B, Wang N, Zheng W. Association between G2385R and R1628P polymorphism of LRRK2 gene and sporadic Parkinson's disease in a Han-Chinese population in South-Eastern China. Neurological Sci. 2013;34(11):2001-6.

23. Fusaki N, Ban H, Nishiyama A, Saeki K, Hasegawa M. Efficient induction of transgene-free human pluripotent stem cells using a vector based on Sendai virus, an RNA virus that does not integrate into the host genome. Proc Japan Acad Series B Phys Biolog Sci. 2009;85(8):348-62.

24. Bhutani K, Nazor KL, Williams R, Tran H, Dai H, Dzakula Z, Cho EH, Pang AWC, Rao M, Cao H, et al. Whole-genome mutational burden analysis of three pluripotency induction methods. Nat Commun. 2016;7:10536.

25. Mandai M, Watanabe A, Kurimoto Y, Hirami Y, Morinaga C, Daimon T, Fujihara M, Akimaru H, Sakai N, Shibata Y, et al. Autologous induced stemcell-derived retinal cells for macular degeneration. New Engl J Med. 2017; 376(11):1038-46.

26. Hsu TR, Sung SH, Chang FP, Yang CF, Liu HC, Lin HY, Huang CK, Gao HJ, Huang $\mathrm{YH}$, Liao HC, et al. Endomyocardial biopsies in patients with left ventricular hypertrophy and a common Chinese later-onset Fabry mutation (IVS4 + 919G > a). Orphanet J Rare Dis. 2014;9:96.

27. Fung $\mathrm{HC}$, Chen $\mathrm{CM}$, Hardy J, Singleton $\mathrm{AB}$, Wu YR. A common genetic factor for Parkinson disease in ethnic Chinese population in Taiwan. BMC Neurol. 2006:6:47.

28. Luo HR, Wu GS, Pakstis AJ, Tong L, Oota H, Kidd KK, Zhang YP. Origin and dispersal of atypical aldehyde dehydrogenase ALDH2487Lys. Gene. 2009; 435(1-2):96-103.

29. Taapken SM, Nisler BS, Newton MA, Sampsell-Barron TL, Leonhard KA, McIntire EM, Montgomery KD. Karotypic abnormalities in human induced pluripotent stem cells and embryonic stem cells. Nat Biotechnol. 2011;29(4): 313-4.

30. Young MA, Larson DE, Sun CW, George DR, Ding L, Miller CA, Lin L, Pawlik KM, Chen K, Fan X, et al. Background mutations in parental cells account for most of the genetic heterogeneity of induced pluripotent stem cells. Cell Stem Cell. 2012;10(5):570-82.

31. Abyzov A, Mariani J, Palejev D, Zhang Y, Haney MS, Tomasini L, Ferrandino AF, Rosenberg Belmaker LA, Szekely A, Wilson M, et al. Somatic copy number mosaicism in human skin revealed by induced pluripotent stem cells. Nature. 2012;492(7429):438-42.

32. Lu J, Li H, Hu M, Sasaki T, Baccei A, Gilbert DM, Liu JS, Collins JJ, Lerou PH. The distribution of genomic variations in human iPSCs is related to replication-timing reorganization during reprogramming. Cell Rep. 2014;7(1): 70-8.

33. Ma H, Morey R, O'Neil RC, He Y, Daughtry B, Schultz MD, Hariharan M, Nery $J R$, Castanon R, Sabatini K, et al. Abnormalities in human pluripotent cells due to reprogramming mechanisms. Nature. 2014;511(7508):177-83.

34. Baghbaderani BA, Syama A, Sivapatham R, Pei Y, Mukherjee O, Fellner T, Zeng $X$, Rao MS. Detailed characterization of human induced pluripotent stem cells manufactured for therapeutic applications. Stem Cell Rev. 2016; 12(4):394-420

35. Amps K, Andrews PW, Anyfantis G, Armstrong L, Avery S, Baharvand H, Baker J, Baker D, Munoz MB, Beil S, et al. Screening ethnically diverse human embryonic stem cells identifies a chromosome 20 minimal amplicon conferring growth advantage. Nat Biotechnol. 2011;29(12):1132-U1113.

36. Vallot $C$, Huret $C$, Lesecque $Y$, Resch A, Oudrhiri N, Bennaceur-Griscelli A, Duret $L$, Rougeulle $C$. XACT, a long noncoding transcript coating the active X chromosome in human pluripotent cells. Nat Genet. 2013;45(3):239-41.

37. Kim JH, Kurtz A, Yuan BZ, Zeng F, Lomax G, Loring JF, Crook J, Ju JH, Clarke $\mathrm{L}$, Inamdar MS, et al. Report of the international stem cell banking initiative workshop activity: current hurdles and Progress in seed-stock banking of human pluripotent stem cells. Stem Cells Transl Med. 2017;6(11):1956-62.

\section{Publisher's Note}

Springer Nature remains neutral with regard to jurisdictional claims in published maps and institutional affiliations.

Ready to submit your research? Choose BMC and benefit from:

- fast, convenient online submission

- thorough peer review by experienced researchers in your field

- rapid publication on acceptance

- support for research data, including large and complex data types

- gold Open Access which fosters wider collaboration and increased citations

- maximum visibility for your research: over $100 \mathrm{M}$ website views per year

At BMC, research is always in progress.

Learn more biomedcentral.com/submissions 